\title{
Human spaceflight: to infertility and beyond
}

\section{Sir,}

Garrett-Bakelman FE et al, demonstrated a spectrum of molecular and physiological changes attributed to spaceflight in their recently published "NASA Twins Study". ${ }^{1}$ During his 340 days in space onboard the International Space Station (ISS), one of a pair of monozygotic twins was not only challenged by noise, isolation, hypoxia, and alterations in the circadian rhythm, but more importantly the exposure to ionizing radiation (IR) and microgravity. Terrestrials are subjected constantly to surface gravity and most if not all physiological processes have adapted accordingly. It is therefore easy to envisage that weightlessness can have consequences for space travellers. $^{2}$

The "NASA Twins Study" focused primarily on the impact of spaceflight on the individual. ${ }^{1}$ However, with plans for significantly longer space missions and ultimately colonization, it is vital to consider multigenerational survival. This therefore places human procreation, under conditions of IR and microgravity, in the spotlight, as it cannot be readily compared with reproduction on Earth. Sperm function and integrity are fundamental to both natural or assisted reproduction, and the current information of spaceflight on mammalian sperm is limited.

Wakayama et al, reported that exposing freeze-dried mouse spermatozoa to space IR (288 days on the ISS at $95^{\circ} \mathrm{C}$ ) did not change the fertilization and birth rates, despite a slight increase in damage to the sperm DNA and male pronuclei. ${ }^{3}$ In contrast, several studies reported not only degenerative changes in seminiferous tubules and severe irreversible damage to the testicular structure of rodents, but also highlighted diminished sperm parameters (e.g., concentration, motility, progressive motility) under conditions of real and simulated microgravity. ${ }^{4-6}$ Furthermore, a decrease in testosterone levels were observed in rats that were onboard COSMOS 2044 or subjected to hindlimb suspension as a model for mimicking microgravity conditions. ${ }^{7,8}$ Thyroid and testicular functions of mice, that spent 91 days onboard the ISS, were also affected. In specific, a significant decrease in androgen receptor expression was observed in their testicular tissue. These changes, related to pituitary control, significantly affected both endocrine homeostasis and reproductive function, both of which could lead to male reproductive dysfunction in space. ${ }^{6}$
Additionally, two dogs that spent 22 days onboard the Kosmos 110 satellite showed increased numbers of spermatozoa with tail curling or absent tail, while rats on Space Lab-3 (7 days) and Cosmos 1887 (13 days) showed a reduction in spermatogonia. ${ }^{9-11}$

Spermatogenesis encompasses one of the most complex and dynamic biological processes. It starts with diploid spermatogonial stem cells that mitotically divide to become spermatocytes, followed by two meiotic divisions resulting in haploid round spermatids that subsequently differentiate into spermatids. The spermatids then undergo spermiogenesis and enter the epididymis to complete maturation and develop progressive motility and the ability to fertilize. During this process, a complex interaction between Sertoli cells and sperm are crucial. Therefore, alterations in the structures and their components caused by the loss of gravity and the effects of the other adverse factors of a space flight could affect the production of spermatozoa with fertilizing capacity.

Of further interest was the increase in somatic cell telomere lengths in the "space twin" as opposed to his brother's telomeres that underwent the normal shortening with replication. Recently, it was shown that sperm telomere length could be associate with fertilization alterations during ICSI procedures. ${ }^{12}$ Unfortunately, the effect of space flight on sperm telomere length was not studied, and the impact of sperm telomere dynamics on the progeny is an added cause of concern.

Many of the reported changes were transient and returned to comparable levels post space flight. However, when future astronauts are faced to "boldly go where no man has gone before -Jean-Luc Picard-", such as visiting Mars (>year) or celestial colonization, the intricate process of gamete production will be exposed synergistically to much longer periods of IR and microgravity. The question remains as to whether the spectrum of biological effects will shift even more and if the impact on natural reproduction would be reversible or become catastrophic.

It is vital to understand the details of these modifications and long-term consequences on male reproductive function. To "Live long and prosper -Spock-", future studies should focus more on these and the development of countermeasures, else the human race might explore themselves to "infertility and beyond". We should ensure that astronauts in future would be able to use the same quote as Darth Vader "I am your father". 
Walter D. Cardona Maya ${ }^{1 *}$, Stefan S. du Plessis ${ }^{2}$

${ }^{1}$ Reproduction Group, Department of Microbiology and Parasitology, Medical School, University of Antioquia, Antioquia, Colombia

${ }^{2}$ Division of Medical Physiology, Faculty of Medicine and Health Sciences, Stellenbosch University,

Tygerberg, South Africa

*Correspondence:

Walter D. Cardona Maya, E-mail: wdario.cardona@udea.edu.co

\section{REFERENCES}

1. Garrett-Bakelman FE, Darshi M, Green SJ, Gur RC, Lin L, Macias BR, et al. The NASA twins study: amultidimensional analysis of a year-long human spaceflight. Science. 2019;364:6436.

2. Lobrich M, Jeggo PA. Hazards of human spaceflight. Science. 2019;364(6436):127-8.

3. Wakayama S, Kamada Y, Yamanaka K, Kohda T, Suzuki H, Shimazu T, et al. Healthy offspring from freeze-dried mouse spermatozoa held on the International Space Station for 9 months. Proc Natl Acad Sci U S A. 2017;114(23):5988-93.

4. Ikeuchi T, Sasaki S, Umemoto Y, Kubota Y, Kubota $\mathrm{H}$, Kaneko $\mathrm{T}$, et al. Human sperm motility in a microgravity environment. Reprod Med Biol. 2005;4(2):161-8.

5. Ding Y, Tang J, Zou J, She R, Wang Y, Yue Z, et al. The effect of microgravity on tissue structure and function of rat testis. Braz $\mathrm{J}$ Med Biol Res. 2011;44(12):1243-50.
6. Masini MA, Albi E, Barmo C, Bonfiglio T, Bruni L, Canesi L, et al. The impact of long-term exposure to space environment on adult mammalian organisms: a study on mouse thyroid and testis. PLoS One. 2012;7(4):e35418.

7. Merrill AH, Wang E, Mullins RE, Grindeland RE, Popova IA. Analyses of plasma for metabolic and hormonal changes in rats flown aboard COSMOS 2044. J Appl Physiol. 1992;73(2 Suppl):132S-5S.

8. Tash JS, Johnson DC, Enders GC. Long-term (6-wk) hindlimb suspension inhibits spermatogenesis in adult male rats. J Appl Physiol. 2002;92(3):1191-8.

9. Fedorova NL. Spermatogenesis in the dogs ugolyok and veterok after the kosmos-110 spaceflight. Kosm Biol Med. 1967;1:28-31.

10. Philpott DE, Sapp W, Williams C, Stevenson J, Black S, Corbett R. Reduction of the spermatogonial population in rat testes flown on Space Lab-3. Physiol. 1985;28(6 Suppl):S211-2.

11. Sapp WJ, Philpott DE, Williams CS, Kato K, Stevenson J, Vasquez M, et al. Effects of spaceflight on the spermatogonial population of rat seminiferous epithelium. FASEB J. 1990;4(1):101-4.

12. Darmishonnejad Z, Tavalaee M, Izadi T, Tanhaei S, Nasr-Esfahani MH. Evaluation of sperm telomere length in infertile men with failed/low fertilization after intracytoplasmic sperm injection. Reprod Biomed Online. 2019;38(4):579-87.

Cite this article as: Cardona Maya WD, du Plessis SS. Human spaceflight: to infertility and beyond. Int J Reprod Contracept Obstet Gynecol 2019;8:5068-9. 